\title{
Structural Rearrangement and Dispersion of Functionalized Graphene Sheets in Aqueous Solutions
}

Yun Jung Lee,,${ }^{\S+}$ Liwei Huang, ${ }^{\natural}$ Howard Wang, ${ }^{,}$Maria L. Sushko, ${ }^{{ }^{*}}$ Birgit Schwenzer, ${ }^{\S}$ Ilhan A. Aksay, "Jun Liu ${ }^{{ }^{*}}$

$\S$ Pacific Northwest National Laboratory, 902 Battelle Boulevard P.O. Box 999, Richland, WA 99352, United States

${ }^{¥}$ Institute for Materials Research and Department of Mechanical Engineering, State

University of New York, Binghamton, NY 13902, United States

" Department of Chemical and Biological Engineering, Princeton University, Princeton, NJ 08544, United States

\section{AUTHOR INFORMATION \\ Corresponding Authors \\ maria.sushko@pnnl.gov, jun.liu@pnnl.gov}

Present Address

${ }^{+}$Department of Energy Engineering, Hanyang University, Seoul 133-791, Korea 


\begin{abstract}
Surfactants are widely used for dispersing graphene and functionalized graphene sheets (FGS) in colloidal suspensions, but there have been few studies of the structure of the dispersed graphene-surfactant complexes in suspension and of their time evolution. Here, we combine experimental study of efficiencies of ionic surfactants/polymers in suspending FGS in water with characterization using atomic force microscopy, small angle neutron scattering, and molecular simulations to probe the detailed structures of FGSs. The small angle scattering technique provides quantitative measurement of structure of graphene sheets in the solution. This study suggests that in both ionic and nonionic surfactants, the dispersion tends to degrade over time either through detachment of the surfactant molecules and structural rearrangements. Ionic surfactants with strong interfacial binding and large molecular weight increase the dispersing power by over an order of magnitude.
\end{abstract}

\title{
Graphical abstract
}

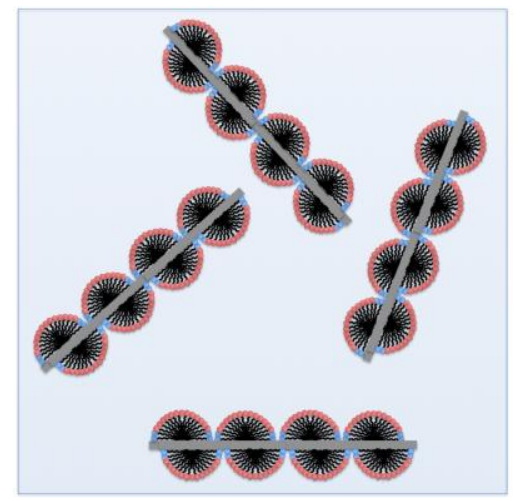

\section{Keywords}

Graphene, dispersion, surfactant, colloidal stability 
As the basal plane of graphite, graphene is a monolayer of carbon atoms tightly packed into a two dimensional (2D) hexagonal crystalline lattice. Interest in this intriguing material has surged after its isolation from graphite as a single sheet about a decade ago. Its outstanding mechanical, electrical, optical, and electrochemical properties along with the high specific surface area have made graphene a promising molecular-scale scaffold or building block for new nanocomposites. While the highest quality graphene is fabricated by "micromechanical cleavage" of graphite, ${ }^{1}$ or by chemical vapor deposition, ${ }^{2}$ large scale production of graphene in bulk relies on graphene oxide extracted from graphite oxide (GO) followed by its reduction towards a pristine graphene-like structure. ${ }^{3}$ Reduction or deoxygenation of graphene oxide is typically accomplished either in the presence of chemical reducing agents ${ }^{4}$ or by rapid thermal heating $^{5}$ of GO. As the reduced graphene oxide sheets by these approaches still contain residual functional groups, we differentiate them from pristine graphene by referring to them as functionalized graphene sheets (FGSs). ${ }^{5}$ We further differentiate between the FGSs based on their oxygen content through the nomenclature $\mathrm{FGS}_{x}$ where $x$ represents the molar carbon to oxygen ratio $(\mathrm{C} / \mathrm{O})$.

Direct exfoliation of graphite by matching its index of refraction with that of the solvent and thus minimizing the Hamaker constant ${ }^{6}$ or in the presence of surfactants ${ }^{7}$ has been pursued as a cost-effective way for preparing colloidal graphene suspensions. ${ }^{8}$ Yet, this is not a viable approach if the choice of dispersion medium is water. ${ }^{8}$ Stable dispersion of graphene in aqueous solutions is often considered critical for many applications, such as preparation of their nanocomposites with oxides. ${ }^{7,9,10}$ However, due to the effect of attractive van der Waals forces, pristine graphene and FGSs will eventually form irreversible agglomerates by restacking to graphitic structures in all solvents, ${ }^{4,11}$ thereby rendering production of homogeneous colloidal 
suspensions of graphene challenging. To prevent agglomeration, electrostatic, steric, and electrosteric stabilization mechanisms, widely used in colloidal science, have been adopted. ${ }^{12,13}$

In addition to the intrinsic functionalization of reduced graphene oxides, graphene has also been functionalized both covalently and non-covalently. Recent approaches of covalent functionalization include 1,3-dipolar cycloaddition, esterification, and functionalization with negatively charged carboxylic or sulfonic groups, for steric and electrostatic stabilization. ${ }^{12}$ Graphene sheets have also been stabilized non-covalently with surfactants/polymers interacting with graphene via hydrophobic, $\pi-\pi$ and ionic interactions. ${ }^{12}$ While some of the surfactants known for dispersing carbon nanotubes (CNTs) were proven effective in dispersing graphene, ${ }^{14}$ others such as Triton® X-100 (TX) reportedly failed to produce stable aqueous dispersions. ${ }^{14}$ Despite many reports of pristine graphene and FGS stabilization in aqueous solutions with surfactants and polymers, obtaining long term stability of the dispersion is still a challenge. There are limited studies on the nature of the dispersed graphene sheets in the solution, their aggregation and structural rearrangement over time. Here we combine experimental study of efficiencies of ionic surfactants/polymers in suspending FGS in water with characterization using atomic force microscopy (AFM), small angle neutron scattering (SANS), and molecular simulations to probe the detailed structure of aqueous FGS dispersions and their structural evolution over time.

FGS dispersions were prepared by horn sonication of their aggregates produced using a thermal expansion method ${ }^{5}$ in $2 \mathrm{w} / \mathrm{v} \%$ aqueous solution of various amphiphilic surfactants or polymers. Compared to the FGSs produced by the chemical reduction of graphene oxide, FGSs prepared by this thermal expansion approach have tunable $\mathrm{C} / \mathrm{O}$ ranging from 7 to 500 , e.g., $\mathrm{FGS}_{7}$ to $\mathrm{FGS}_{500}{ }^{5}$ rendering their conductivity and hydrophobicity also adjustable. In this study, we 
limited our work only to $\mathrm{FGS}_{14}$, as it is the most widely used form for bulk scale applications. The chemical structures of surfactants and polymers used to assist the dispersion of $\mathrm{FGS}_{14}$ (in general, referred to as FGS from hereon) in water are given in Table 1. The efficiencies of surfactants in suspending FGS were measured and compared, as the dispersed FGS concentration $(\mathrm{mg} / 100 \mathrm{~mL})$ left in the supernatant after centrifugation of the horn-sonicated suspension. Surfactant mass contents, determined by thermo gravimetric analysis (TGA), were excluded from the mass of FGS paper to determine the mass of the dispersed FGS.

Table 1. Chemical structures of surfactants and polymers used for dispersing FGS in water.

Sodium dodecyl sulfate [SDS]

The presence of individual graphene sheets was confirmed by tapping mode AFM imaging of different dispersions (Fig. 1a). All the samples were prepared by dip-coating of the surfactant mediated dispersions of FGS on silicon substrates. Surfactants were removed by calcination at $500{ }^{\circ} \mathrm{C}$. Figure 1a shows AFM images of flat graphene flakes with a thickness of $\sim 1 \mathrm{~nm}$, which 
corresponds to the thickness of single graphene sheets. Lateral dimensions range from several hundred nanometers to $\sim 1 \mu \mathrm{m}$. The dispersions were further characterized by UV-Vis spectroscopy. The absorption spectrum of surfactant dispersed FGS 14 showed a peak at $275 \mathrm{~nm}$ with a broad background (Figure $1 \mathrm{~b}$ inset) that is consistent with the previously reported result for graphene aqueous dispersions. ${ }^{4}$ Optical absorbance per unit length $(\mathrm{A} / \mathrm{l})$ at $660 \mathrm{~nm}$ was measured and plotted for serial dilutions of dispersions. All dispersions tested show LambertBeer behavior (Figure 1b) giving absorption coefficient of $\alpha_{660}=5880 \mathrm{Lg}^{-1} \mathrm{~m}^{-1}$.
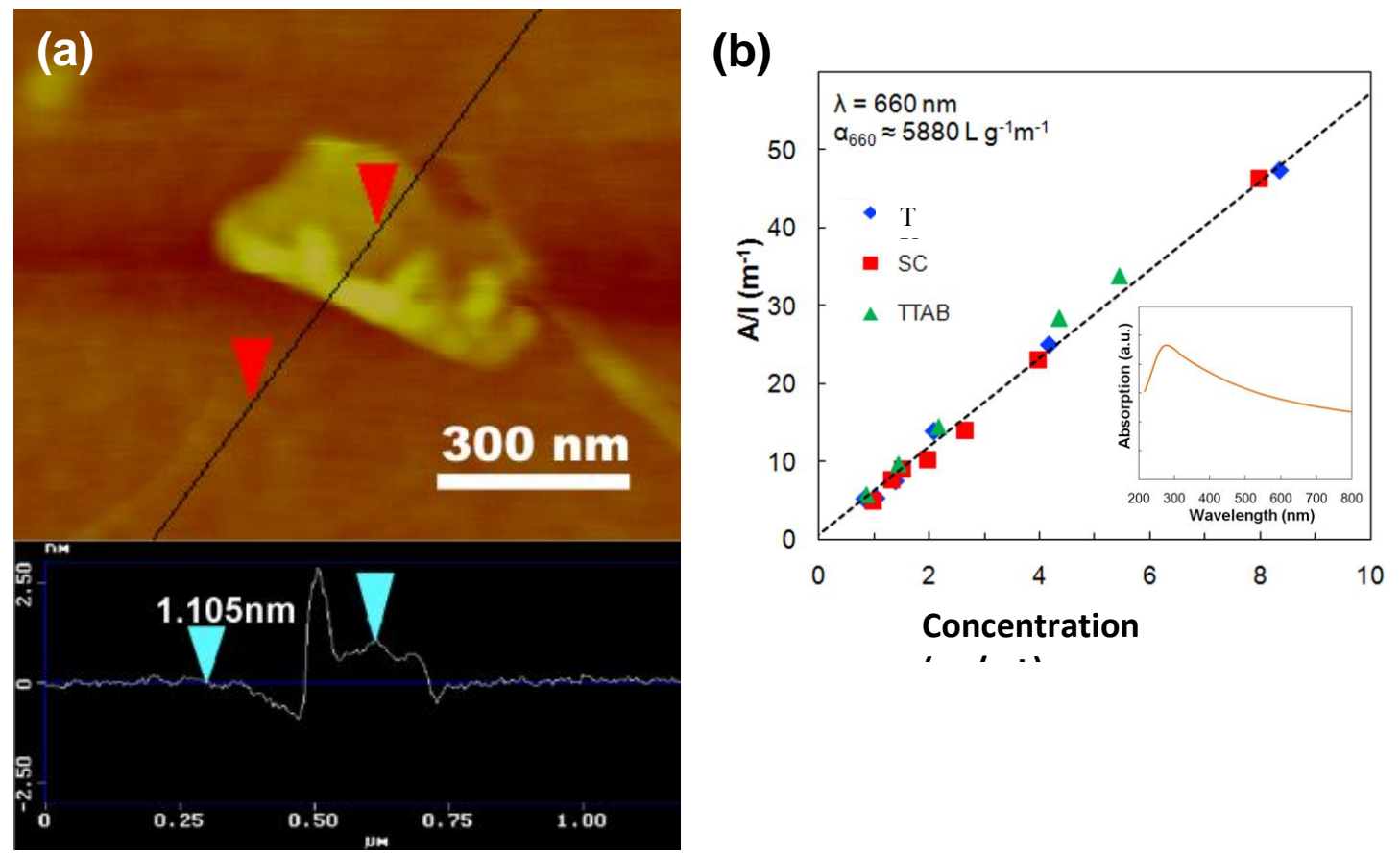

Figure 1. (a) AFM image of $\mathrm{FGS}_{14}$ suspended with $\mathrm{SC}$ with line scan height profile. (b) Optical absorbance per unit length (A/l) at $660 \mathrm{~nm}$ as a function of $\mathrm{FGS}_{14}$ concentration in various aqueous surfactant solutions. Inset: typical absorption spectrum of $\mathrm{FGS}_{14}$ dispersion.

Several ionic and non-ionic surfactants were compared. The surfactant dispersion efficiency is defined as the concentration of dispersed FGS in horn sonicated suspension of 200 $\mathrm{mg}$ of $\mathrm{FGS}_{14}$ powder in $200 \mathrm{~mL}$ of $2 \mathrm{wt} \%$ aqueous surfactant solution (see Supporting information). It follows from Table 2 that the non-ionic surfactant TX-100 is not as efficient in 
dispersing FGS as the ionic surfactants. Furthermore, sodium cholate (SC) with planar $\pi-\pi$ structures stands out among the anionic surfactant providing high FGS dispersion efficiency. This result is consistent with the literature reports that many molecules with planar hydrophobic regions such as polystyrene, pyrene, perylene, and amino acid or sodium cholate ${ }^{7}$ yield stable graphene dispersions, while sodium dodecylsulfate (SDS) with a short hydrocarbon chain was found inefficient in producing stable aqueous dispersion of graphitic nanoplates in full coverage regime. ${ }^{14}$ These differences suggest that strong interactions with the graphene surface are important for good dispersion. SC interacts with graphene by forming a monolayer with hydrophilic charged head-group exposed in solution and the planar region in direct contact with graphene to maximize the strength of van der Waals interactions with the substrate.

Table 2. Surfactant and polymer efficiencies in dispersing $\mathrm{FGS}_{14}$ in water.

\begin{tabular}{|c|c|c|c|c|}
\hline & $\begin{array}{l}\text { Surfactant/ } \\
\text { polymer }^{(a)}\end{array}$ & Molecular weight & $\begin{array}{c}\text { Surface charge } \\
\text { density of surfactant } \\
\text { layer on FGS } \\
\left(\mathrm{e} / \mathbf{n m}^{2}\right)\end{array}$ & $\begin{array}{c}\text { Dispersed FGS } \\
\text { concentration } \\
(\mathrm{mg} / \mathbf{1 0 0 m L})\end{array}$ \\
\hline \multirow{5}{*}{ anionic } & SDS & 288.4 & 1.275 & 3.55 \\
\hline & SDSA & 272.4 & 1.3 & 3.82 \\
\hline & SDBS & 348.5 & 1.2 & 3.29 \\
\hline & & 430.6 & 1.5 & 7.19 \\
\hline & CLEVIOS ${ }^{\mathrm{TM}}$ & $\begin{array}{l}\text { Solid content } \\
\sim 1.3 \%\end{array}$ & $1.8-2.2$ & 44.7 \\
\hline \multirow{2}{*}{ cationic } & TTAB & 336.4 & 1.4 & 3.93 \\
\hline & CTAB & 364.5 & 1.7 & 8.15 \\
\hline non-ionic & Triton $®$ X 100 & 647 & 0 & 2.94 \\
\hline
\end{tabular}

(a) Surfactant concentration is $2 \mathrm{w} / \mathrm{v} \%$

According to experimental and molecular dynamics data the density of SC monolayer on graphene is 1.53 molecule/nm ${ }^{2}{ }^{15}$ For linear hydrocarbon molecules, the strength of van der Waals interactions with the substrate scales with chain length, suggesting that longer molecules 
should have stronger interfacial binding and higher graphene dispersion efficiency. The structure of surfactant layer is another factor that influences graphene dispersion efficiency as evidenced by the differences in the performance of SDS in dispersing carbon nanotubes and graphene. To elucidate this aspect, molecular simulations were performed for a series of linear surfactants of the type $C_{m} X$, where $C_{m}$ stands for a hydrocarbon chain of $m$ carbons and $X$ stands for a charged head-group. For example, for SDS $m=12$ and $X=\mathrm{SO}_{4}{ }^{-}$. In agreement with experimental ${ }^{16}$ and molecular dynamics ${ }^{17}$ data, simulations showed that at full coverage $C_{m} X$ surfactants with $12 \leq m<18$ form hemicylindrical micelles on graphene surface and have a characteristic average density distribution of the charged head-groups as shown in Figure 2. First sharp peak on the density distribution corresponds to the molecules lying flat on the surface and the second broader peak to the adjacent molecules. According to the corresponding distribution of counterions, the charge on these groups is almost fully compensated by condensed counterions. As a result, these groups do not contribute to the overall surface charge density of the surfactant layer. Furthermore, while the radius of the hemicylindrical micelles scales with surfactant chain length, the position of the first two peaks on the density distribution remains the same (Fig. 2).

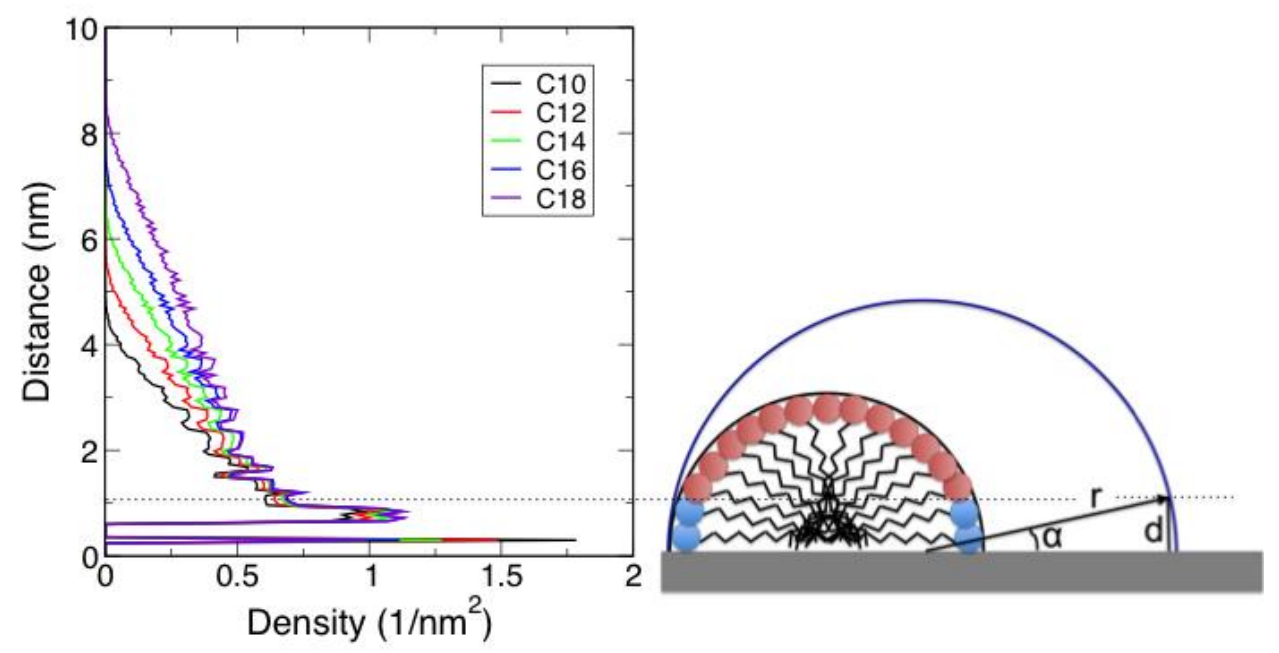

Figure 2. Simulated chain length dependence of the density of charged head groups of $C_{m} X$ surfactants. The schematic shows the side view of hemicylindrical micelles for two different $m$. 
Charged groups are shown as red spheres and those compensated by condensed counterions as blue spheres.

As schematically shown in Figure 2, the fraction of the exposed surfactant charged groups in each hemicylindrical micelle varies monotonically with chain length and can be calculated as $f=(\pi-2 \alpha) / \pi$, where $\sin \alpha=d / r$ with $d$ and $r$ being the thickness of the electrically neutral surfactant layer and micelle radius, respectively. The second factor defining the effective surface charge density is the maximum surface coverage or the density of surfactant molecules on graphene surface. Simulations show that surface coverage increases with chain length due to stronger chain-chain and chain-substrate van der Waals interactions until it reaches the maximum at C16 (Table 3). The resulting effective charge density of surfactant layer increases with surfactant chain length and saturates at $m=16$ with maximum charge density of $1.7 \mathrm{e} / \mathrm{nm}^{2}$ (Table 3). The surface charge density of other ionic surfactants and polymers studied in this work was reported in the literature

Table 3. Correlation between the structure of $C_{m} X$ surfactant layers on graphene and the effective charge density.

(a) this work and ref.

\begin{tabular}{|c|c|c|c|}
\hline$C_{m} X$ & $\begin{array}{l}\text { Height of } \\
\text { surfactant } \\
\text { layer (nm) }\end{array}$ & $\begin{array}{c}\text { Surface } \\
\text { coverage } \\
\left(\mathbf{m o l e c u l e} / \mathbf{n m}^{2}\right)\end{array}$ & $\begin{array}{l}\text { Surface } \\
\text { charge } \\
\text { density } \\
\left(\mathrm{e} / \mathbf{n m}^{2}\right)\end{array}$ \\
\hline C12 & 3.92 & 1.5 & 1.3 \\
\hline C14 & 4.50 & 1.6 & 1.4 \\
\hline C16 & 5.07 & $1.9^{(\mathrm{a})}$ & 1.7 \\
\hline C18 & 5.92 & 1.9 & 1.7 \\
\hline
\end{tabular}

or can be estimated based on the chemical composition. Combining these data with the measured FGS dispersion efficiency shows a clear correlation between the surface charge density of 
surfactant layers, defining the strength of long-range repulsive electrostatic interactions between the FGSs, and the concentration of dispersed FGS (Table 2). It is noteworthy, that on highly curved surfaces, such as carbon nanotubes, $C_{m} X$ surfactants form a cylindrical shell around the substrate with all the charged tail-groups exposed in solution. ${ }^{19}$ As a result, the effective surface charge density of the SDS layer on carbon nanotube is almost 15\% larger than that on graphene, which explains the differences in the corresponding dispersion efficiencies. Moreover, in the low coverage regime when the concentration of SDS is well below critical surface aggregation concentration and in the $40-60 \mu \mathrm{M}$ range SDS molecules form dense planar monolayer on $\mathrm{FGS}^{11}$ with the average density $1.5-2.0$ molecule $/ \mathrm{nm}^{2}$ or surface charge density of $1.5-2.0$ $\mathrm{e} / \mathrm{nm}^{2}{ }^{20}$ In this regime, SDS also produces stable FGS dispersions through electrostatic stabilization mechanism and the concentration of dispersed FGS is as high as $10 \mathrm{mg} / 100 \mathrm{~mL}{ }^{11}$ These data suggest that colloidal stability of FGS in the presence of ionic surfactants is dominated by mean-field electrostatics and depend on the effective surface charge density of surfactant layers on FGS. However, short-range interactions due to steric, hydration and ion mediated second order electrostatic interactions, sensitive to the details of the distribution of discrete charges on the surface, were reported to give significant contribution to the total repulsive interactions between FGSs at separation smaller than $1.5 \mathrm{~nm} .{ }^{21}$ These forces are expected to play the major role in time evolution of FGS dispersions since they define the energy barriers for the aggregation and restacking of FGSs. ${ }^{22}$

The relative role of electrostatic and non-electrostatic forces in the long-term stability of FGS aqueous dispersions was examined using SANS. The approach previously applied to semiquantitatively assess the degree of dispersion of single- and multi-walled carbon nanotubes was employed. $^{23,24}$ The power law dependence of the total scattering cross-section (which is 
proportional to the recorded scattering intensity, $I$ ) over momentum transfer wave vector, $Q$, with a power exponent $\alpha\left(Q^{-\alpha}\right)$ gives a semi-quantitative assessment of the degree of dispersion based on simple geometrical scaling laws. For dilute dispersions of rigid rods such as shortened single wall CNTs, $I$ follows the $Q^{-1}$ power law. ${ }^{23,24}$ Graphene sheets are flexible 2D objects; however, surfactant decorated FGSs in solution are expected to be locally semi-rigid. Then, close to $Q^{-2}$ power law should be followed for good dispersion. Deviation from the $Q^{-2}$ power suggests a more complex structure than simple graphene sheets (folding or aggregation).

SANS spectra of the representative FGS dispersions in ionic (SC) and non-ionic (TX) surfactant solutions containing $0.10 \%$ and $0.04 \%$ of FGS by mass, respectively, both freshly prepared (labeled as "fresh") and the same solutions stored in ambience for 27 days (labeled as "stored"), are shown in Figure 3. SANS spectra of neat surfactant solutions, 5\% by mass in deuterated water are shown in the inset. The symbols are the experimental data and solid curves are model calculations. The as-prepared FGS suspensions could be well described by a power law with an incoherent background, $I(Q)=I_{0} / Q^{\alpha}+I_{\text {inc }}$, where both the coefficient, $I_{0}$, and the incoherent scattering intensity, $I_{\text {inc }}$, are constant. The best fits show power exponents of $2.33 \pm$ 0.01 and $2.72 \pm 0.01$ for as prepared FGS/SC and FGS/TX solutions, respectively. The FGS/SC suspension has better dispersion as implied by the closer to $Q^{-2}$ power law behavior, whereas the Triton X-100 suspension is inferior, which is consistent with the apparent dispersion power of the two surfactants as shown in Table 2. The higher exponent value suggests a more complex structure than the simple 2D sheets. 


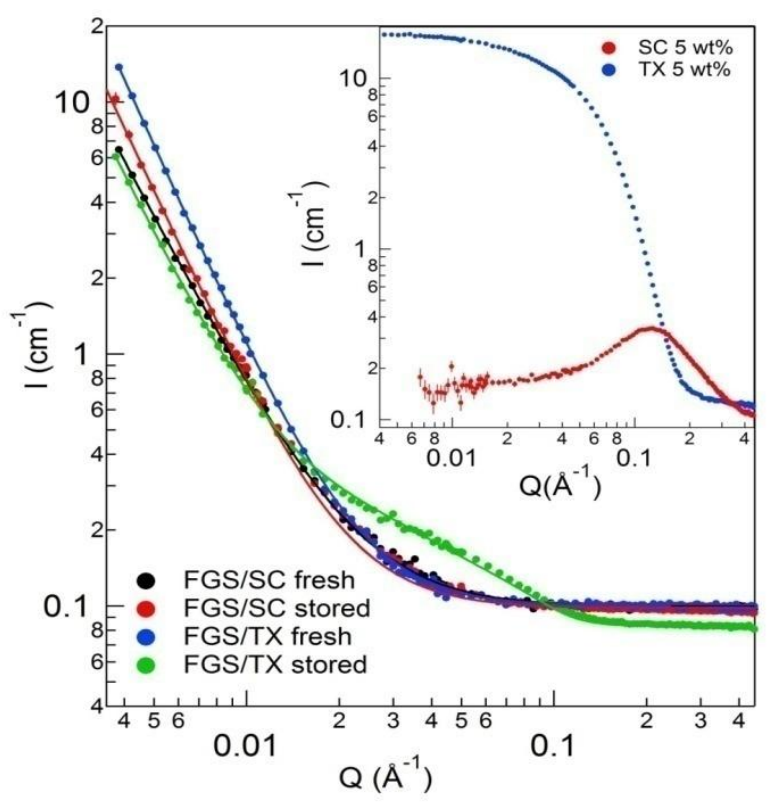

Figure 3. SANS spectra of freshly prepared FGS/SC and FGS/TX suspension in $\mathrm{D}_{2} \mathrm{O}$ (labeled with "fresh") and the same suspensions stored in ambience for 27 days (labeled with "stored"). The symbols are experimental data and the curves through the symbols are best fitting. The inset shows the spectra of neat SC and TX solutions with $5 \%$ surfactant by mass in $\mathrm{D}_{2} \mathrm{O}$.

After 27 days of storage under ambient conditions, the FGS/TX suspension shows visible agglomeration and sedimentation, while the FGS/SC suspension remains visually indistinguishable from the as-prepared one. However, the SANS spectra of the stored suspensions show apparent deviations from the as-prepared ones for both. For the stored FGS/TX suspension, there is excess scattering in the $Q$-range of $0.01-0.1 \AA^{-1}$, which may be attributed to surfactant desorption from FGSs and the formation of micelles in solution. As shown in the inset, TX micelles are non-interacting so that the total scattering intensity scales with the individual micelle scattering function by the surfactant concentration, whereas SC solution shows a strong correlation peaks at $\sim 0.13 \AA^{-1}$ due to long-range charge interactions. Hence, the spectra of FGS/TX after storage could be modeled as the sum of power-law scattering from the remaining dispersed FGS in solution, the free TX micelles scattering following the same scattering function as in Figure 3 inset, and the incoherent scattering. Best fit produces a 
power exponent of $2.54 \pm 0.01$ and free surfactant concentration of $0.044 \%$ by mass in the form of micelles. The incoherent scattering, which is proportional to the total surfactant amount in the neutron beam path, decreases from $(0.100 \pm 0.002) \mathrm{cm}^{-1}$ for the as-prepared solution to $(0.082 \pm$ $0.002) \mathrm{cm}^{-1}$ for the stored one, implying $18 \%$ of TX surfactants fall out of the beam with FGS sedimentation, while those remaining in solution appear to have better dispersion than in the freshly prepared solution upon the storage-induced self-fractionation. This change is likely to be due to the sedimentation of the more aggregated graphene sheets during storage.

The spectrum of the stored FGS/SC suspension was evaluated with the power law scattering and a constant background. Although deviations between the experimental data and the fitted curve are observed at the intermediate $Q \sim 0.02 \AA^{-1}$ caused by a small amount of excess scattering from free surfactant in the solution, it does not affect the accurate assessment of the power law scaling at low $Q$ and of the incoherent background at high $Q$. The power exponent of $2.64 \pm 0.02$ indicates slight degradation of the dispersion after storage, while the incoherent scattering of $(0.098 \pm 0.004) \mathrm{cm}^{-1}$ is statistically identical to $(0.099 \pm 0.001) \mathrm{cm}^{-1}$ for the asprepared solution, indicating no measurable precipitation in the FGS/SC solution after 27 days' storage. Although the FGS/SC suspension shows slight degradation over 27 days, it still demonstrates better stability than the FGS/TX suspension against surfactant desorption and sedimentation.

These data further highlight the importance of strong attractive interactions between FGS and surfactant hydrophobic region and strong long-range repulsive interactions between surfactant layers decorating neighboring FGSs for a long-term stability of FGS dispersion. Compared to SC, TX has weaker attractive interactions between tert-octylphenyl tail and FGS and shorter-range repulsive interactions between polyethylene oxide head-groups, which have 
steric and hydration nature. The combination of these two factors facilitates TX surfactant sharing between adjacent FGSs followed by surfactant expulsion from the gap between graphene sheets overtime and partial restacking of graphene sheets (schematically shown in Fig. S2).

The results from current study have important implications for practical applications. First, in suspensions where the surfactants do not strongly bind to graphene, such as ordinary ionic surfactants, the surfactant may detach from the graphene surface during aging and cause graphene restacking and eventual sedimentation of the graphene aggregates. Even for some good dispersing surfactant such as SC, the surfactant will not detach from the graphene surface, but over time structural degradation and some degree of agglomeration still occurs. This may be due to the fact that the graphene sheets are too hydrophobic and the long-range van der Waals forces are too strong. To overcome such limitations, the surfactants need to not only bind to graphene, but also need to provide long-range electrostatic or steric repulsion. Preliminary studies suggest surfactants such as CLEVIOS ${ }^{\mathrm{TM}} \mathrm{P}$ (PEDOT:PSS) can have exceptional dispersion power to fulfill these needs. These polymers are known to form large core-shell particles 10-30 nm in diameter in aqueous solution, which can incorporate graphene sheets in their hydrophobic core. ${ }^{25}$ PEDOT:PSS effectively shields direct graphene-graphene van der Waals interactions and the concentration of the dispersed FGS is then defined by graphene uptake by each PEDOT:PSS particle and by the concentration of these particles in solution. More systematic study of the effect of the molecular weight and the chain chemistry is underway and will be reported in the future.

\section{Acknowledgment}

This research is supported by the U.S. Department of Energy (DOE), Office of Basic Energy Sciences, Division of Materials Sciences and Engineering under Award KC020105-FWP12152. 
Pacific Northwest National Laboratory (PNNL) is a multiprogram national laboratory operated for DOE by Battelle. HW acknowledges the support of NSF grant CMMI-0928865 and the National Institute of Standards and Technology, US Department of Commerce, for providing neutron scattering facilities for this study. Simulations were performed using PNNL Institutional Computing at PNNL.

\section{References}

(1) Novoselov, KS, D Jiang, F Schedin, TJ Booth, VV Khotkevich, SV Morozov, AK Geim. 2005. "Two-dimensional atomic crystals". Proceedings of the National Academy of Sciences of the United States of America, 102, 10451-10453.

(2) Kim, KS, Y Zhao, H Jang, SY Lee, JM Kim, KS Kim, JH Ahn, P Kim, JY Choi, BH Hong. 2009. "Large-scale pattern growth of graphene films for stretchable transparent electrodes". Nature, 457, 706-710.

(3) Park, S, RS Ruoff. 2009. "Chemical methods for the production of graphenes". Nature Nanotechnology, 4, 217-224.

(4) Li, D, MB Muller, S Gilje, RB Kaner, GG Wallace. 2008. "Processable aqueous dispersions of graphene nanosheets". Nature Nanotechnology, 3, 101-105.

(5) McAllister, MJ, JL Li, DH Adamson, HC Schniepp, AA Abdala, J Liu, M Herrera-Alonso, DL Milius, R Car, RK Prud'homme, IA Aksay. 2007. "Single sheet functionalized graphene by oxidation and thermal expansion of graphite". Chemistry of Materials, 19, 4396-4404.

(6) Hamilton, CE, JR Lomeda, ZZ Sun, JM Tour, AR Barron. 2009. "High-yield organic dispersions of unfunctionalized graphene". Nano Letters, 9, 3460-3462.

(7) Lotya, M, PJ King, U Khan, S De, JN Coleman. 2010. "High-concentration, surfactant-stabilized graphene dispersions". Acs Nano, 4, 3155-3162.

(8) Coleman, JN. 2009. "Liquid-phase exfoliation of nanotubes and graphene". Advanced Functional Materials, 19, 3680-3695.

(9) Li, XL, W Qi, DH Mei, ML Sushko, I Aksay, J Liu. 2012. "Functionalized graphene sheets as molecular templates for controlled nucleation and self-assembly of metal oxide-graphene nanocomposites". Advanced Materials, 24, 5136-5141.

(10) Wang, DH, R Kou, D Choi, ZG Yang, ZM Nie, J Li, LV Saraf, DH Hu, JG Zhang, GL Graff, J Liu, MA Pope, IA Aksay. 2010. "Ternary self-assembly of ordered metal oxide-graphene nanocomposites for electrochemical energy storage". Acs Nano, 4, 1587-1595.

(11) Hsieh, AG, S Korkut, C Punckt, IA Aksay. 2013. "Dispersion stability of functionalized graphene in aqueous sodium dodecyl sulfate solutions". Langmuir, 29, 1483114838 .

(12) Texter, J. 2014. "Graphene dispersions". Current Opinion in Colloid \& Interface Science, $19,163-174$. 
(13) Georgakilas, V, M Otyepka, AB Bourlinos, V Chandra, N Kim, KC Kemp, P Hobza, R Zboril, KS Kim. 2012. "Functionalization of graphene: Covalent and non-covalent approaches, derivatives and applications". Chemical Reviews, 112, 6156-6214.

(14) Stankovich, S, RD Piner, XQ Chen, NQ Wu, ST Nguyen, RS Ruoff. 2006. "Stable aqueous dispersions of graphitic nanoplatelets via the reduction of exfoliated graphite oxide in the presence of poly(sodium 4-styrenesulfonate)". Journal of Materials Chemistry, 16, $155-158$.

(15) Sim, Y, J Park, YJ Kim, MJ Seong, S Hong. 2011. "Synthesis of graphene layers using graphite dispersion in aqueous surfactant solutions". Journal of the Korean Physical Society, 58, 938-942.

(16) Wanless, EJ, WA Ducker. 1996. "Organization of sodium dodecyl sulfate at the graphite-solution interface". Journal of Physical Chemistry, 100, 3207-3214.

(17) Tummala, NR, A Striolo. 2008. "Role of counterion condensation in the selfassembly of sds surfactants at the water-graphite interface". Journal of Physical Chemistry B, 112, 1987-2000.

(18) Bie, YQ, J Horng, ZW Shi, L Ju, Q Zhou, A Zettl, DP Yu, F Wang. 2015. "Vibrational spectroscopy at electrolyte/electrode interfaces with graphene gratings". Nature Communications, 6, 7593.

(19) Uddin, NM, FM Capaldi, B Farouk. 2012. "Molecular dynamics simulations of carbon nanotube dispersions in water: Effects of nanotube length, diameter, chirality and surfactant structures". Computational Materials Science, 53, 133-144.

(20) Sammalkorpi, M, AZ Panagiotopoulos, M Haataja. 2008. "Structure and dynamics of surfactant and hydrocarbon aggregates on graphite: A molecular dynamics simulation study". Journal of Physical Chemistry B, 112, 2915-2921.

(21) Wu, B, XN Yang. 2013. "Molecular simulation of electrolyte-induced interfacial interaction between sds/graphene assemblies". Journal of Physical Chemistry C, 117, 2321623223.

(22) Yang, P, F Liu. 2014. "Understanding graphene production by ionic surfactant exfoliation: A molecular dynamics simulation study". Journal of Applied Physics, 116.

(23) Wang, H, W Zhou, DL Ho, KI Winey, JE Fischer, CJ Glinka, EK Hobbie. 2004. "Dispersing single-walled carbon nanotubes with surfactants: A small angle neutron scattering study". Nano Letters, 4, 1789-1793.

(24) Wang, H. 2009. "Dispersing carbon nanotubes using surfactants". Current Opinion in Colloid \& Interface Science, 14, 364-371.

(25) Dehsari, HS, EK Shalamzari, JN Gavgani, FA Taromi, S Ghanbary. 2014. "Efficient preparation of ultralarge graphene oxide using a pedot:Pss/go composite layer as hole transport layer in polymer-based optoelectronic devices". Rsc Advances, 4, 55067-55076. 